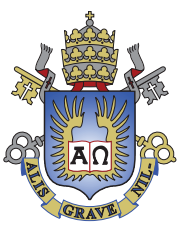

Rafael Magri

Nonlinear Error Correction
Models: Estimation and
Testing

Dissertação de Mestrado DEPARTAMENTO DE ECONOMIA Postgraduate Program in Economics 
Rafael Magri

\section{Nonlinear Error Correction Models: Estimation and Testing}

Dissertation presented to the Postgraduate Program in Economics of the Departamento de Economia, PUC-Rio as partial fulfillment of the requirements for the degree of Mestre em Economia.

Advisor: Prof. Marcelo C. Medeiros 
Dissertation presented to the Postgraduate Program in Economics of the Departmento de Economia, PUC-Rio as partial fulfillment of the requirements for the degree of Mestre em Economia. Approved by the following commission:

\author{
Prof. Marcelo C. Medeiros \\ Advisor \\ Departamento de Economia - PUC-Rio
}

Prof. João Victor Issler

EPGE

Prof. Adrian Pizzinga

IAPUC

Prof. José Eugenio Leal

Coordinator of the Centro Técnico Científico da PUC-Rio 
All rights reserved.

\section{Rafael Magri}

Rafael Magri graduated from Universidade de Brasília (UnB) in Economics.

Bibliographic data

Magri, Rafael

Nonlinear Error Correction Models: Estimation and Testing / Rafael Magri; orientador: Marcelo C. Medeiros. -2010 .

40 f.: il.; $30 \mathrm{~cm}$

Dissertação (Mestrado em Economia) - Pontifícia Universidade Católica do Rio de Janeiro, Rio de Janeiro, 2010.

Inclui bibliografia.

1. Economia - Teses. 2. Modelo de Correção de Erros; 3. Não-linearidade;. 4. Expansão de Taylor.. I. Medeiros, Marcelo C.. II. Pontifícia Universidade Católica do Rio de Janeiro. Departamento de Economia. III. Título. 


\section{Acknowledgments}

To my adviser, Marcelo C. Medeiros.

To CAPES and Instituto Allis, for the financial support. 


\section{Resumo}

Magri, Rafael; Medeiros, Marcelo C.. Modelos de Correção

de Erro Não-Lineares: Estimação e Teste. Rio de Janeiro, 2010. 40p. Dissertação de Mestrado - Departamento de Economia, Pontifícia Universidade Católica do Rio de Janeiro.

Testes existentes para não-linearidade em Modelos de Correção de Erros são altamente intensivos computacionalmente e apresentam parâmetros de estorvo na distribuição assintótica, que precisam ser levantadas através de simulações por bootstrap. É proposto um teste consistente, implementável em qualquer pacote estatístico e que apresenta distribuição assintótica QuiQuadrado. Além disso, experimentos de Monte Carlo mostram que em pequena amostra o teste tem boas propriedades de tamanho e poder, muitas vezes melhores do que os testes existentes. Também é apresentada uma condição sob a qual um estimador em dois estágios para os parâmetros do modelo é assintoticamente normal. A aplicação do modelo a preços internacionais de commodities agrícolas mostra evidência de ajuste nãolinear nos preços de trigo.

\section{Palavras-chave}

Modelo de Correção de Erros; Não-linearidade; Expansão de Taylor. 


\section{Abstract}

Magri, Rafael; Medeiros, Marcelo C.. Nonlinear Error Correction Models: Estimation and Testing. Rio de Janeiro, 2010. 40p. MsC Dissertation — Departamento de Economia, Pontifícia Universidade Católica do Rio de Janeiro.

Existing tests for nonlinearity in vector error correction models are highly intensive computationally and have nuisance parameters in the asymptotic distribution, what calls for cumbersome bootstrap calculations in order to assess the distribution. Our work proposes a consistent test which is implementable in any statistical package and has Chi-Squared asymptotics. Moreover, Monte Carlo experiments show that in small samples our test has nice size and power properties, often better than the preexisting tests. We also provide a condition under which a consistent two step estimator for the model parameters is asymptotically normal. Application to international agricultural commodities prices show evidence of nonlinear adjustment to the long run equilibrium on the wheat prices.

\section{Keywords}

Vector Error Correction Model; Nonlinearity; Taylor Expansion. 


\section{Contents}

1 Introduction $\quad \mathbf{1 0}$

2 The Model $\quad 13$

$\begin{array}{lll}3 & \text { Testing Linearity } & 17\end{array}$

4 Estimation and Asymptotic Properties 20

$5 \quad$ Small Sample Properties $\quad 22$

6 Application: International Commodities Prices $\quad 25$

$\begin{array}{lll}7 & \text { Conclusions } & 30\end{array}$

8 Appendix $\quad 34$

8.1 Propositions 34

仓 8.2 Lemmas 38 


\section{List of Figures}

2.1 Transition Functions 15

$\begin{array}{lll}2.2 & \text { Adjustment Functions } & 16\end{array}$

6.1 Adjustment functions 27

6.2 Estimated Adjustment Functions 29 


\section{List of Tables}

5.1 EMPIRICAL SizE. 23

5.2 EMPIRICAL POWER. 24

5.3 EMPIRICAL POWER. 24

6.1 Linearity Tests. 26

6.2 Linear ECM Estimations for Wheat Prices 26

6.3 Nonlinear ECM Estimations for Wheat Prices 28 